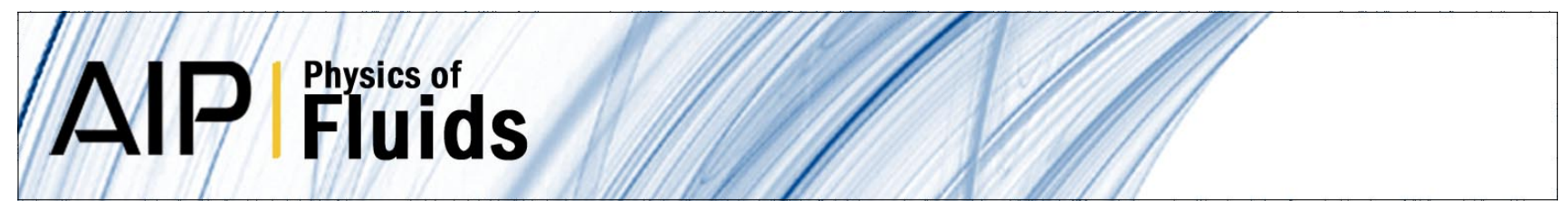

\title{
Liquid film drag out in the presence of molecular forces
}

I. Schmidhalter, R. L. Cerro, M. D. Giavedoni, and F. A. Saita

Citation: Phys. Fluids 25, 032105 (2013); doi: 10.1063/1.4794972

View online: http://dx.doi.org/10.1063/1.4794972

View Table of Contents: http://pof.aip.org/resource/1/PHFLE6/v25/i3

Published by the American Institute of Physics.

\section{Additional information on Phys. Fluids}

Journal Homepage: http://pof.aip.org/

Journal Information: http://pof.aip.org/about/about_the_journal

Top downloads: http://pof.aip.org/features/most_downloaded

Information for Authors: http://pof.aip.org/authors

\section{ADVERTISEMENT}

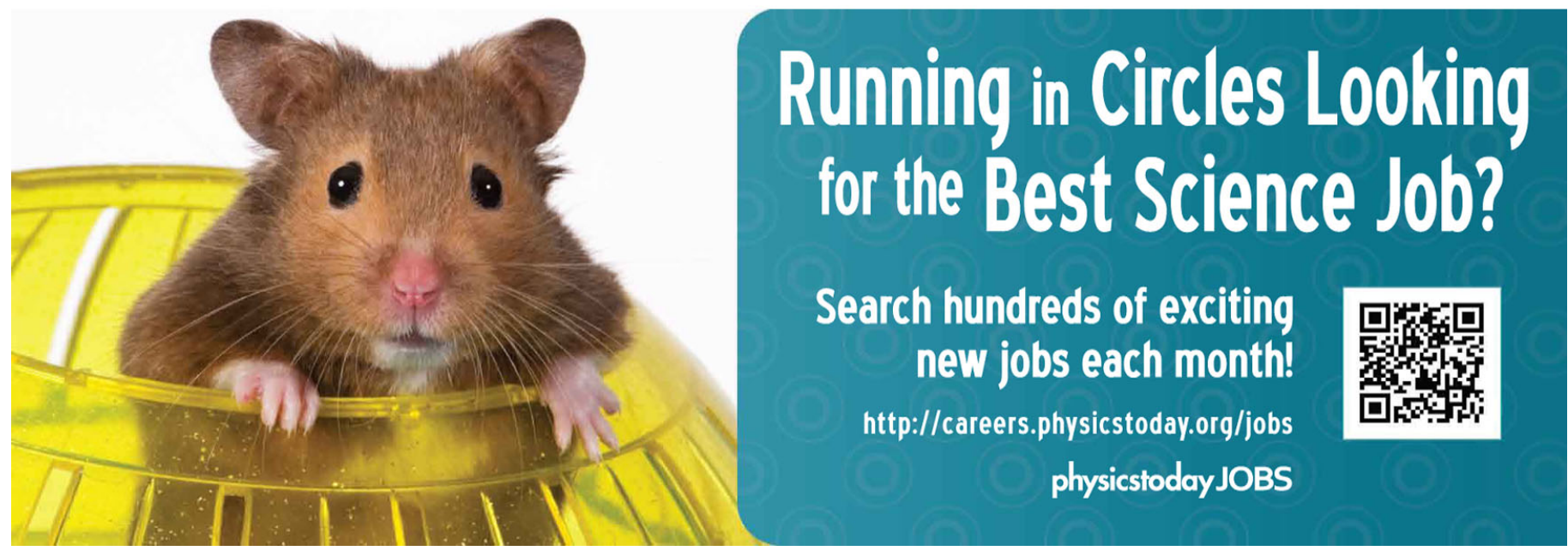




\title{
Liquid film drag out in the presence of molecular forces
}

\author{
I. Schmidhalter, ${ }^{1}$ R. L. Cerro, ${ }^{2}$ M. D. Giavedoni, ${ }^{1, a)}$ and F. A. Saita ${ }^{1}$ \\ ${ }^{1}$ INTEC (CONICET-UNL), Güemes 3450, Santa Fe 3000, Argentina \\ ${ }^{2}$ Department of Chemical and Materials Engineering, University of Alabama in Huntsville, \\ Huntsville, Alabama 35899, USA
}

(Received 3 August 2012; accepted 13 February 2013; published online 18 March 2013)

\begin{abstract}
From a practical as well as a conceptual point of view, one of the most interesting problems of physicochemical hydrodynamics is the drag out of a liquid film by a moving solid out of a pool of liquid. The basic problem, sometimes denoted the Landau-Levich problem [L. Landau and B. Levich, "Dragging of a liquid by a moving plate," Acta Physicochim. USSR 17, 42-54 (1942)], involves an interesting blend of capillary and viscous forces plus a matching of the static solution for capillary rise with a numerical solution of the film evolution equation, neglecting gravity, on the downstream region of the flow field. The original solution describes experimental data for a wide range of Capillary numbers but fails to match results for large and very small Capillary numbers. Molecular level forces are introduced to create an augmented version of the film evolution equation to show the effect of van der Waals forces at the lower range of Capillary numbers. A closed form solution for static capillary rise, including molecular forces, was matched with a numerical solution of the augmented film evolution equation in the dynamic meniscus region. Molecular forces do not sensibly modify the static capillary rise region, since film thicknesses are larger than the range of influence of van der Waals forces, but are determinant in shaping the downstream dynamic meniscus of the very thin liquid films. As expected, a quantitatively different level of disjoining pressure for different values of molecular constants remains in the very thin liquid film far downstream. Computational results for a wide range of Capillary numbers and Hamaker constants show a clear transition towards a region where the film thickness becomes independent of the coating speed. (c) 2013 American Institute of Physics. [http://dx.doi.org/10.1063/1.4794972]
\end{abstract}

\section{INTRODUCTION}

Dip coating is a widely known process in which a solid is withdrawn with constant velocity out of a liquid bath in order to deposit a film of uniform thickness. When the system is free of surface active agents, and operates at intermediate removal speeds, the driving force for liquid entrainment is viscosity while gravity promotes drainage. The simple hydrodynamic problem does not have a unique solution unless capillary forces are introduced to determine film thickness downstream. The original solution introduced by Landau and Levich $(\mathrm{L} \& \mathrm{~L})^{1}$ assumes that at low velocities the meniscus can be split in two regions: a thin region called the dynamic meniscus where the interface profile is slightly curved, and the static region which is slightly perturbed by the motion of the solid plate (see Fig. 1). Since the dynamic meniscus is nearly flat, the main flow is in the upward direction, and the interface shape results from the competition between viscous and surface forces (gravity effect is neglected in their analysis). A main assumption of the $\mathrm{L}_{\mathrm{L}} \mathrm{L}^{1}$ approach requires matching the curvature of the static meniscus at the point where the interface becomes parallel to the solid, with the constant curvature of the dynamic meniscus. At the point of matching, the curvature of the

\footnotetext{
a) Author to whom correspondence should be addressed. Electronic mail: madelia@santafe-conicet.gov.ar.

1070-6631/2013/25(3)/032105/13/\$30.00 25, 032105-1 $\quad$ C 2013 American Institute of Physics
} 


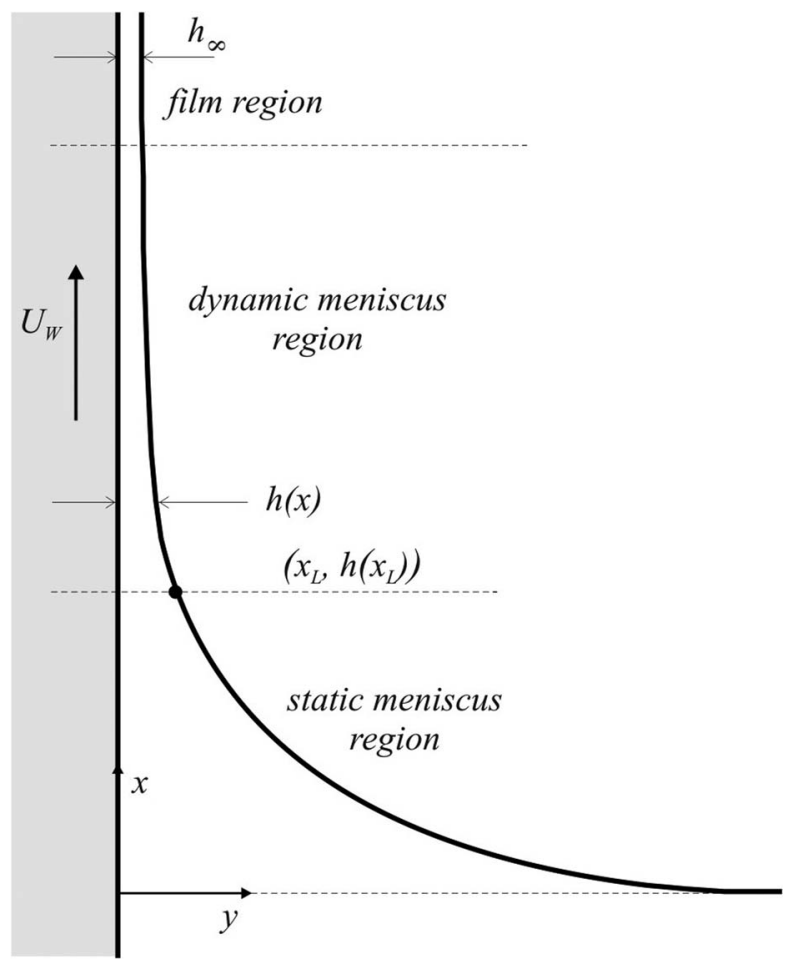

FIG. 1. Geometrical sketch of flow field due to the vertical removal of a flat solid from a viscous liquid pool.

static meniscus and the curvature of the dynamic meniscus reach a maximum while the angle of inclination of the interface in the static meniscus side is equal to zero.

The flat plate moves upward out of a liquid bath at a constant velocity, $u=U_{w}$. The liquid properties (viscosity, $\mu$, density, $\rho$, and surface tension, $\sigma$ ), are constant; shear from the surrounding air is neglected and its pressure is arbitrarily set equal to zero. Following $L \& L^{1}$ analysis, we identify three domain regions: the static meniscus, the dynamic meniscus, and the flat film region. In the flat film region, viscous, gravity, and inter-molecular forces are balanced. For very thin films, gravity is negligible and the velocity profile is near plug-flow and velocity is assumed to be everywhere equal to the substrate velocity. At the lower region near the liquid pool, the liquid interface raising from the pool is assumed to be independent of the motion of the solid such that the shape of the interface is determined by gravity and surface tension only (i.e., it is the shape of capillary rise). In the intermediate dynamic meniscus region, the curvature of the interface is determined by integration of the film evolution equation and matched to the static shape. The magnitude of the surface curvature is subsequently used to determine flow rate and liquid film thickness in the downstream flat film region. Apparently unaware of the Landau-Levich ${ }^{1}$ paper, van Rossum ${ }^{2}$ published an experimental paper where the velocity profile far downstream in the flat film region includes gravity forces and determines a parabolic drainage profile

$$
\begin{aligned}
& u(x, y)=U_{w}+\left(\frac{1}{\mu} \frac{d p}{d x}+\frac{\rho g}{\mu}\right)\left[\frac{y^{2}}{2}-y h\right] ; \quad q=\int_{y=0}^{y=h_{\infty}} u d y=U_{w} h_{\infty}-\left(\frac{\rho g h_{\infty}^{3}}{3 \mu}\right), \\
& \text { Landau-Levich: }{ }^{1} h_{\infty} \cong \frac{q}{U_{w}}, H=\frac{h(x)}{h_{\infty}}, \\
& \text { Van Rossum: }{ }^{2} Q=\frac{q}{U_{w}}\left(\frac{\rho g}{\mu U_{w}}\right)^{1 / 2}=T-\frac{T^{3}}{3} \Leftrightarrow T=h_{\infty}\left(\frac{\rho g}{\mu U_{w}}\right)^{1 / 2},
\end{aligned}
$$




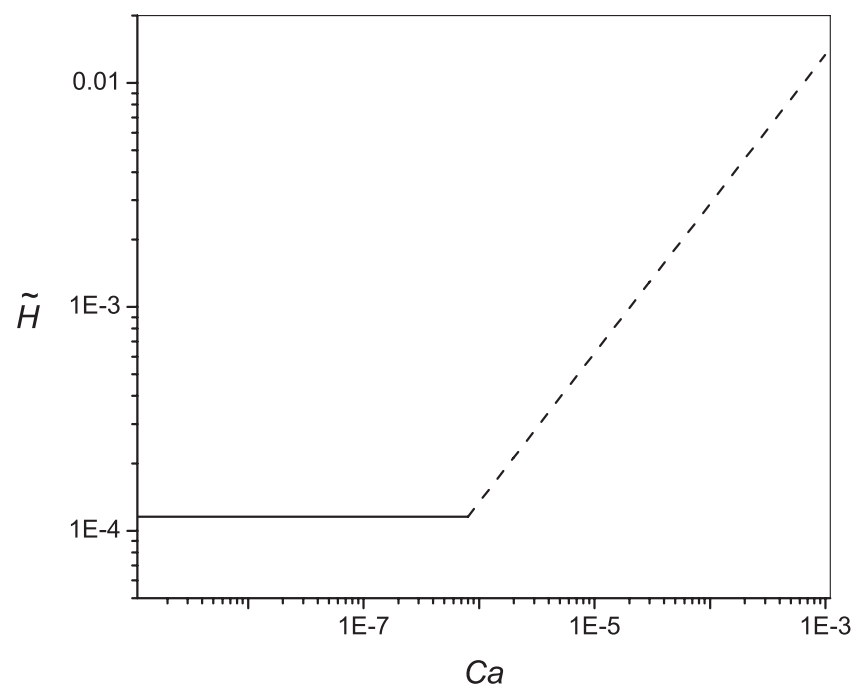

FIG. 2. Schematic representation of the experiments by Quéré et al. ${ }^{6}$ showing dimensionless film thickness versus dimensionless velocities for a small cylindrical fiber.

where $h_{\infty}$ is the film thickness far downstream and its definition assumes a uniform velocity profile. The $L \& L^{1}$ problem was analyzed thoroughly and valuable experimental data generated by the late Professor Tallmadge ${ }^{3,4}$ and students. Based on the definition of film thickness introduced by van Rossum, ${ }^{2}$ Wilson ${ }^{5}$ performed a rigorous analysis of the drag out problem recognizing the presence of two characteristic lengths, the capillary length, $L_{C}=\sqrt{\sigma / \rho g}$, used to render interface curvature dimensionless and a film thickness length resulting from the ratio of the square root of the Capillary number and the capillary length, $d=\left(\mu U_{w} / \rho g\right)^{1 / 2}$. Wilson's ${ }^{5}$ analysis resulted in a formal description of the drag out problem including gravity, but did not bring any new theoretical results for vertical withdrawal.

The Landau-Levich ${ }^{1}$ solution delivers a dimensionless expression for asymptotic film thickness as a function of Capillary number

$$
\hat{H}(x \rightarrow \infty)=\frac{q}{U_{w} L_{C}}=0.93 C a^{2 / 3} ; \quad C a=\frac{\mu U_{w}}{\sigma} .
$$

The functional relationship described by Eq. (2) is valid for a wide range of Capillary numbers, roughly from $10^{-6}<C a<10^{-1}$, but fails to describe the regions of very small, $\mathrm{Ca}<10^{-6}$, or large, $\mathrm{Ca}>10^{-1}$, Capillary numbers.

Quéré et al. ${ }^{6}$ experimentally studied the dip coating of fibers of small radius at very low velocities. Their results, schematically represented in Figure 2, show that the coated thickness measured in units of the radius of the fiber $(\tilde{H})$ departs from Landau and Levich law (dashed line) at very small withdrawal velocities; in fact, at low Capillary numbers the film thickness becomes independent of the coating speed (solid line).

At large Capillary numbers, inertial forces become important and dimensionless film thickness levels off at a constant value. The limit of large Capillary numbers was analyzed by Cerro and Scriven ${ }^{7}$ based on a rapid-flow integral formulation of the film evolution equation. The large Capillary limit results in a rational expression for the dimensionless film thickness

$$
Q=\frac{q}{U_{w}}\left(\frac{\rho g}{\mu U_{w}}\right)^{1 / 2}=\left[\frac{6^{1 / 2}-1}{2 \cdot 6^{1 / 2}}\right]^{1 / 2}=0.5439 \cdots
$$

There have been a few attempts to analyze the very small Capillary numbers limit. Diaz Martin ${ }^{8}$ extended the treatment first introduced by Teletzke, ${ }^{9}$ developing an augmented film evolution equation with the addition of a disjoining pressure term resulting from van der Waals molecular-level forces. Although the goal of Diaz Martin ${ }^{8}$ was to demonstrate the effect of molecular and Marangoni 
forces on Langmuir-Blodgett film deposition, her computations include numerical solutions of the film evolution equation for a wide range of Capillary numbers and Hamaker constants. Krechetnikov and Homsy, ${ }^{10}$ performed an asymptotic matching of static profile and dynamic film including an electrostatic potential. Recently, ${ }^{11}$ a detailed theoretical analysis was performed including the ad hoc electrostatic potential ${ }^{10}$ as well as a Debye-Huckel potential.

An interesting and extended numerical analysis of dip coating was done by Schunk et al. ${ }^{12}$ where drying and mass transfer effects were included on their shaping of $L \& L^{1}$ films as well as the related Langmuir-Blodgett and sol-gel depositions.

In Sec. II, we develop the augmented film evolution equation, based on the Landau-Levich ${ }^{1}$ formulation, plus the introduction of a disjoining pressure term resulting from van der Waals forces. In Sec. III, we describe the static meniscus region using the augmented Young-Laplace equation. In Sec. IV, we perform computational solutions of the augmented film evolution equation. Analysis and conclusions are described in Sec. V.

\section{THE AUGMENTED FILM EVOLUTION EQUATION}

The development of the film evolution equation follows the guidelines formalized by Higgins and Scriven ${ }^{13}$ where the $y$-component of the equation of motion is integrated to obtain pressure, the derivative of pressure is inserted in the $x$-component of the equation of motion and the resulting differential equation is integrated once across the film thickness, $0 \leq y \leq h(x)$. The augmented version of the film evolution equation ${ }^{9}$ includes a disjoining pressure term created by molecular forces (Note: the word disjoining is used here generically for disjoining/conjoining fields). In this work, the liquid phase is constituted by non-polar hydrocarbon molecular species and molecular forces are represented by van der Waals forces using a truncated form of the 6-12 Lennard-Jones potential. ${ }^{14}$ Throughout the domain of flow defined for this problem, the starting point is the augmented film evolution equation in dimensional form

$$
\left(h-h_{\infty}\right)-\left(\frac{h^{3}-h_{\infty}^{3}}{3}\right)\left(\frac{\rho g}{\mu U_{w}}\right)-\frac{h_{m}^{2}}{h}\left(\frac{\sigma}{\mu U_{w}}\right) \frac{d h}{d x}-\frac{h^{3}\left(\sigma / \mu U_{w}\right)}{3} \frac{d^{2} \sin \theta}{d x^{2}}=0,
$$

where the first term in Eq. (4) is the viscous contribution, the second term is the gravity force contribution, the third term is the disjoining pressure resulting from molecular forces, and the last term is the capillary pressure term. The interface for capillary rise on a flat solid surface is two dimensional, i.e., the horizontal curvature is everywhere zero. The derivative of $\sin \theta$, where $\theta$ is the angle of inclination of the interface with respect to the solid surface, is an exact expression for vertical curvature. The characteristic molecular thickness, $h_{m}$, is typical of capillary problems with molecular forces (Diaz Martin et al. ${ }^{14}$ ) and is defined as

$$
h_{m}^{2}=\frac{A_{L L}^{[6]} G_{6}\left(\theta_{0}\right)-A_{S L}^{[6]}}{6 \pi \sigma},
$$

where $A_{L L}^{[6]}$ and $A_{S L}^{[6]}$ are the liquid/liquid and solid/liquid Hamaker constants, respectively, for a truncated [6-12] Lennard-Jones potential. The angular function corrects the liquid/liquid contribution for the angle of inclination of the interface with respect to the solid surface. In all our computations, the angle of inclination of the interface at the point of matching was zero, as a consequence we can safely take $G_{6}(0)=1$. Equations (4) and (5) are developed in Diaz Martin et al. ${ }^{14}$

We will use Eq. (4) to obtain the equations that describe static capillary rise and dynamic film evolution phenomena. Neglecting viscous forces, the augmented Young-Laplace equation is recovered from Eq. (4). If on the other hand gravity is neglected, the film evolution equation is recovered. Without the gravity term and molecular forces, Eq. (4) is identical to the equation used by $L \& L^{1}$ in their analysis. Neglecting gravity, the dimensionless version of Eq. (4) includes only one dimensionless parameter

$$
\frac{d^{3} H}{d X^{3}}=\frac{3 \alpha}{H^{4}} \frac{d H}{d X}-3\left(\frac{H-1}{H^{3}}\right) ; \quad \alpha=\frac{h_{m}^{2}}{h_{\infty}^{2} C a^{2 / 3}},
$$


where the dimensionless film thickness is defined on the basis of the downstream film thickness, $H$ $=h / h_{\infty}$, and the dimensionless coordinate, $X=x \mathrm{Ca}^{1 / 3} / h_{\infty}$ includes the Capillary number. Notice that the expression for the curvature of the interface is now an approximation where $d h / d x=1$ is used to simplify meniscus curvature. This dimensionless form of the film evolution equation is consistent with the $L \& L^{1}$ approach where the definition of the approximate plug flow profile is used to characterize both film thickness and flow rate. Equation (6) has two distinct advantages for computation, (1) it has only one parameter, $\alpha$, and (2) the downstream limit of the dimensionless film thickness has a known and simple value, $H(X \rightarrow \infty)=1$.

Following Landau-Levich, ${ }^{1}$ we assume that the flow field has three distinctive regions, (1) a static meniscus region that can be described, in our case, by the augmented Young-Laplace equation, (2) a dynamic meniscus region whose interfacial profile, obtained by integrating Eq. (6), joins the static meniscus at the maximum curvature of this one, and (3) a downstream uniform film region that will be used as the starting point of our dynamic computations. In other words, below the maximum point of curvature of the meniscus, the shape of the air/liquid interface is described by the augmented Young-Laplace equation that is a capillary-gravity shape including disjoining pressure. Above the point of maximum curvature, the shape of the interface is described by the augmented film evolution equation, Eq. (6).

\section{THE STATIC MENISCUS REGION}

Neglecting viscous forces and considering $\left(h_{\infty} / h\right)^{3} \ll 1$ in the static meniscus, the augmented film evolution equation, Eq. (4), can be integrated once between the liquid pool (i.e., $x=0$, $2 H=0, h \rightarrow \infty)$ to recover the augmented Young-Laplace equation describing capillary rise on a flat, vertical surface

$$
-\frac{d}{d x} \sin \theta=2 H=\frac{x}{L_{C}^{2}}-\frac{h_{m}^{2}}{h^{3}} .
$$

Equation (7) can be easily integrated exactly, to get an expression for the static contact angle as a function of capillary and molecular forces

$$
1-\sin \theta_{0}=\frac{x_{L}^{2}}{2 L_{C}^{2}}-\int_{x=0}^{x_{L}} \frac{h_{m}^{2}}{h^{3}} d x .
$$

Note that at $x=0$, the angle of inclination of the free surface is $\pi / 2$; in Eq. (8) $\theta_{0}$ is the static macroscopic contact angle, and $x_{L}$ is the point of maximum capillary rise. Neglecting molecular force contributions, Eq. (8) reduces to the well-known relationship for the static contact angle in capillary rise developed by McNutt and Andes. ${ }^{15}$ One of the key assumptions of the $\mathrm{L} \& \mathrm{~L}^{1}$ approach is that under drag out conditions the contact angle is zero. This assumption is valid as long as the film thickness at the point of matching is larger than a few tens of nanometers (Diaz Martin et al. ${ }^{16}$ ). For the same reason, the contribution of molecular forces in Eq. (8) is very small; thus, we neglect them to compute the value of $x_{L}$. At the point of maximum capillary rise, $x_{L}$, the curvature of the interface is obtained substituting the value of $x_{L}$ into Eq. (7)

$$
\left.2 H\right|_{x_{L}}=\frac{\sqrt{2}}{L_{C}}-\frac{h_{m}^{2}}{h^{3}\left(x_{L}\right)} .
$$

Equation (9), i.e., the curvature of the static meniscus at $x_{L}$, is multiplied by $h_{\infty} / C a^{2 / 3}$ to put it into the dimensionless form employed for the evolution equation; then, it is equated to the constant value of the curvature $(\beta)$ provided by Eq. (6). The result is

$$
\left.2 H\right|_{x_{L}} \frac{h_{\infty}}{C a^{2 / 3}}=\left[\frac{\sqrt{2}}{L_{C}}-\frac{h_{m}^{2}}{h^{3}\left(x_{L}\right)}\right] \frac{h_{\infty}}{C a^{2 / 3}}=\beta .
$$

In order to find the value of the constant curvature, $L \& L^{1}$ integrated numerically their simplified version of the film evolution equation. In our case, we must integrate numerically the dimensionless 
TABLE I. Values of physicochemical constants for n-heptane/PTFE system.

\begin{tabular}{lcc}
\hline \hline$\sigma(\mathrm{N} / \mathrm{m})$ & & $L_{C}(\mathrm{~m})$ \\
0.0203 & & 0.00174 \\
$A_{S L}(\mathrm{~J})$ & $A_{L L} \cdot G_{6}(\mathrm{~J})$ & $h_{m}(\mathrm{~m})$ \\
$4.030 \times 10^{-20}$ & $4.296 \times 10^{-20}$ & $8.34 \times 10^{-11}$ \\
\hline \hline
\end{tabular}

form of our augmented version [Eq. (6)]. Numerical integration of this expression returns the dimensionless curvature at the point of matching, $\beta$. Equation (10) together with the expression of $\alpha$ provides two equations from which $h_{\infty}$ and $C a$ can be obtained since $h(x)$ is also knownas $H(x) / h_{\infty}$-from the integration of Eq. (6). Our computed predictions were obtained in this way; although, for the $\mathrm{Ca}$ values here explored a similar result would have been obtained by not considering the term $h_{m}{ }^{2} / h^{3}\left(x_{L}\right)$ in Eq. (10) because it is negligible compared with $\sqrt{2} / L_{C}$. With the purpose of showing the rightness of our assertion, we compare our computed predictions for the n-heptane/polytetrafluoroethylene (PTFE) system with the values of $h_{\infty}$ obtained from Eq. (11) which is just a rearrangement of Eq. (10) without the molecular term

$$
h_{\infty}=\frac{\beta C a^{2 / 3}}{\sqrt{2} / L_{C}} .
$$

Table I gives the values of the physicochemical constants for a system of heptane over PTFE.

Table II gives values of the dimensionless curvature at the point of matching, $\beta$, and the film thickness at the point of matching plus values of film thickness far downstream, $h_{\infty}$, obtained from Eq. (11) and from our computations.

Agreement between theoretical and computational values of the equilibrium film thickness far downstream indicates that molecular forces have little or no effect on configuring the shape of the static meniscus. Indeed, the term including molecular thickness and film thickness at the point of matching is two to four orders of magnitude smaller than the term including capillary length; i.e., $h_{m}^{2} / h^{3}\left(x_{L}\right) \ll \sqrt{2} / L_{C}$. Film thicknesses at the point of matching are very small and difficult to detect and measure experimentally, $10^{-6} \mathrm{~m} \leq h(x) \leq 10^{-7} \mathrm{~m}$, but very large when compared with molecular thickness, $h_{m}=8.34 \times 10^{-11} \mathrm{~m}$. Also, film thicknesses at the points of matching, $h\left(x_{L}\right)$, shown in Table II, are much larger than downstream film thicknesses, $h_{\infty}$; therefore, the larger contribution of disjoining pressure is at the dynamic meniscus region downstream the matching point. We could expect that for extremely small Capillary numbers, when film thickness at the point of matching is below a few tens of nanometers, $h\left(x_{L}\right)<50 \times 10^{-9} \mathrm{~m}$, the projection of the solution of the augmented Young-Laplace equation could yield contact angles larger than zero at that point, ${ }^{16}$ and in such case the static meniscus region also will be affected by molecular-level forces.

\section{NUMERICAL INTEGRATION OF THE AUGMENTED FILM EVOLUTION EQUATION}

Equation (6) can be integrated using a backward shooting method, starting far downstream where the thickness of the film, $H(X \rightarrow \infty)=1$. The first and second derivatives of film thickness are subject to a small perturbation parameter and integration proceeds using the MATLAB function ODE45. Integration was stopped when the curvature of the film approaches a constant value. The condition of constant curvature was verified by computing the third derivative of $H(X)$ with respect

TABLE II. Computed values of $\beta$ and $h$ at the point of matching, and final film thicknesses, calculated [ $h_{\infty}$ Eq. (11)] and computed [ $h_{\infty}$ (Comp)], for selected values of $C a$.

\begin{tabular}{lllll}
\hline \hline $\mathrm{Ca}$ & $\beta$ & $h\left(x_{L}\right)(\mathrm{m})$ & $h_{\infty}(\mathrm{m})$ Eq. $(11)$ & $h_{\infty}(\mathrm{m})(\mathrm{Comp})$ \\
\hline $10^{-6}$ & 1.3213 & $2.7686 \times 10^{-6}$ & $1.6257 \times 10^{-7}$ & $1.6257 \times 10^{-7}$ \\
$10^{-7}$ & 1.4413 & $6.6685 \times 10^{-7}$ & $3.8207 \times 10^{-8}$ & $3.8205 \times 10^{-8}$ \\
$10^{-8}$ & 3.81168 & $4.0201 \times 10^{-7}$ & $2.1771 \times 10^{-8}$ & $2.1768 \times 10^{-8}$ \\
\hline \hline
\end{tabular}




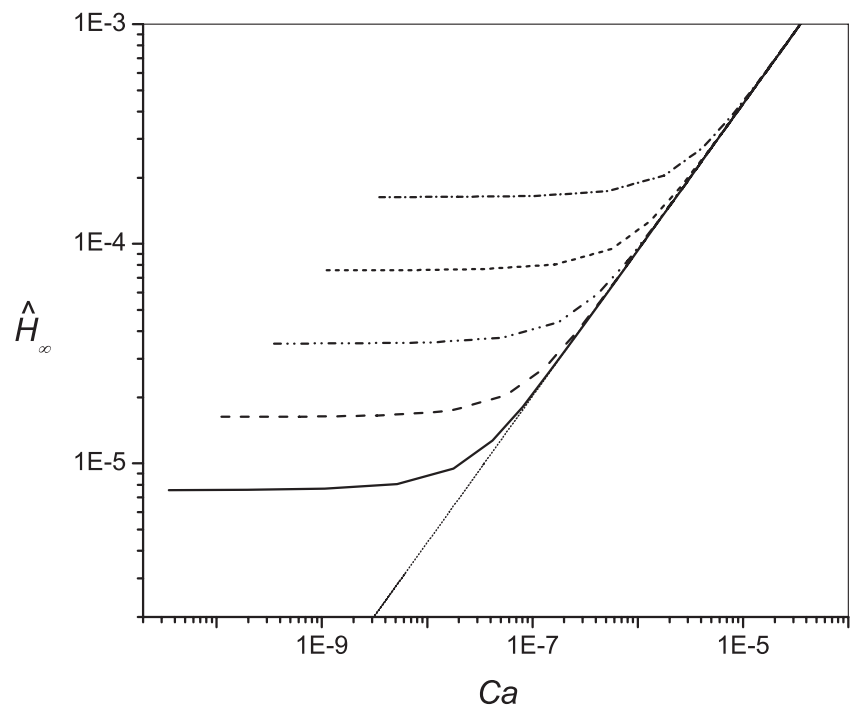

FIG. 3. Log-log plot of dimensionless film thickness versus Capillary number for A equal to $6.13 \times 10^{-16}$ (一), 6.13 $\times 10^{-15}(--), 6.13 \times 10^{-14}(-\cdots), 6.13 \times 10^{-13}(---)$, and $6.13 \times 10^{-12}(-\cdot)$. The dotted line is the ${\mathrm{L} \& \mathrm{~L}^{1}}^{1}$ solution.

to $X$ and using a termination point where $d^{3} H / d X^{3} \leq 10^{-2}$. The matching condition specifies that the point of maximum curvature of the static region and the point of constant curvature of the dynamic meniscus region must be the same

$$
\left(\frac{d^{2} H}{d X^{2}}\right)_{H \rightarrow 0}^{\text {static }}=\left(\frac{d^{2} H}{d X^{2}}\right)_{H \rightarrow \infty}^{\text {dynamic }}
$$

The first set of computations were completed for intermediate Capillary numbers without molecular forces and results were compared with previous numerical computations using the full 2D Navier-Stokes equations and a finite element computational method. ${ }^{17}$ In all cases, film thicknesses computed using the film evolution equation were within $2 \%$ of film thickness computed using the finite element method.

As it was done by L\&L, ${ }^{1}$ computations of the film evolution equation within the dynamic meniscus region are performed using a dimensionless form for the film thickness, $H(X)=h(x) / h_{\infty}$. To compare our results with $L \& L^{1}$ results (Eq. (2)) and to highlight departures from these results at very small Capillary numbers, dimensionless film thickness and dimensionless molecular parameters were redefined using capillary length, $L_{C}$, as the characteristic length value

$$
\hat{H}=\frac{h}{L_{C}}=H \frac{q}{U_{w} L_{C}} ; \quad \mathrm{A}=\frac{h_{m}^{2}}{L_{C}^{2}}=\frac{A_{L L}^{[6]}-A_{S L}^{[6]}}{6 \pi \sigma L_{C}^{2}} .
$$

One of the most important findings from these computations is that dimensionless film thickness, $\hat{H}_{\infty}$ reaches a constant value at a Capillary number that depends on the value of the dimensionless parameter A. These results are shown in Figure 3 in a $\log -\log$ plot of $\hat{H}_{\infty}$ vs $\mathrm{Ca}$ for characteristic constant values of $\mathrm{A}$.

To cover a wider range, the range of molecular forces described by the molecular parameter, A, is purposely larger than typical values for non-polar liquids on partially wetting solid surface (see, for example, Tables at Diaz Martin et al. ${ }^{14}$ ). For values of $h_{m}=10^{-10} \mathrm{~m}$, similar to the one already shown for the n-heptane/PTFE system, at $10^{-7} \sim \mathrm{Ca} \sim 10^{-6}$, dimensionless film thicknesses depart from the $\mathrm{L} \& \mathrm{~L}^{1}$ solution and approach a constant asymptotic value of $\hat{H}_{\infty} \approx 2 \times 10^{-5}$. This dimensionless value corresponds to actual film thickness of $h_{\infty} \leq 3.8 \times 10^{-8} \mathrm{~m}$. This is the range of film thicknesses where one can expect molecular forces to be of the same order of magnitude than capillary forces but with different sign, thus forcing a thicker downstream film thickness. 
Experimental confirmation of these results is difficult for at least two reasons; (1) the film is too thin to be detected by conventional optical methods since film thickness is below the wavelength of visible light, and (2) a film with such small thickness would evaporate rapidly to achieve thermodynamic equilibrium with surrounding air. Quéré et al. ${ }^{6}$ determined, via an indirect method, film thicknesses of dodecane on fibers of a few micrometers in diameter. The experimental results of Quéré et al. ${ }^{6}$ are schematically represented in Figure 2. Although the systems are not directly comparable because the large effect of the second fundamental curvature in fibers, their experimental data show an identical trend for the film thickness to remain constant below a certain removal speed.

The curves illustrated in Figure (3) strongly suggest that the film thickness can be correlated with the molecular parameter, A, when it becomes independent of the Capillary number. In fact, a $\log -\log$ plot of $\hat{H}_{\infty}$ in the region where it becomes constant, as a function of A (not shown here) gives a straight line, and a linear fit of the data gives the following expression:

$$
\hat{H}_{\infty}=\frac{h_{\infty}}{L_{C}}=0.89 \cdot \mathrm{A}^{1 / 3} .
$$

The foregoing equation can also be obtained from Eq. (11) and the definition of $\alpha$, as follows. Since we are in the region where $\hat{H}_{\infty}$ becomes constant regardless of the Capillary value, Eq. (11) and the definition of $\alpha$ might be rewritten as

$$
\begin{gathered}
\beta C a^{2 / 3}=\frac{h_{\infty} \sqrt{2}}{L_{C}}, \\
\alpha C a^{2 / 3}=\left(\frac{h_{m}}{h_{\infty}}\right)^{2} .
\end{gathered}
$$

The terms on the right of Eq. (15) are all constants; then, the ratio between them gives another constant

$$
\frac{\beta}{\alpha}=\frac{h_{\infty} \sqrt{2}}{L_{C}\left(h_{m} / h_{\infty}\right)^{2}}=K^{3} ;
$$

from expression (16) $h_{\infty} / L_{C}=K(\mathrm{~A} / \sqrt{2})^{1 / 3}$ or $h_{\infty} / L_{C}=K 0.891(\mathrm{~A})^{1 / 3}$, which would be exactly Eq. (14) if $K \rightarrow 1$ (i.e., $\beta / \alpha \rightarrow 1$ ) when $C a \rightarrow 0$. That this is the case can be envisaged by integrating Eq. (6) from the film zone $(H=1$ at $X \rightarrow \infty)$ up to the matching point with the static meniscus $\left(H=H^{*}\right.$ at $\left.X=X^{*}\right)$; this integration gives

$$
\frac{d^{2} H}{d X^{2}}=\beta=\alpha\left(1-\frac{1}{H^{* 3}}\right)-\int_{X \rightarrow \infty}^{X^{*}} \frac{H-1}{H^{3}} d X
$$

The first term on the right of Eq. (17) represents the molecular forces in the dynamic meniscus and it is approximately $\alpha$ since $H^{*}$ is much larger than one. The second term represents the viscous forces in the same region and it is the integration of a function whose value is zero at the film zone, reaches a maximum when $H=1.5$ and decreases to zero again as the matching zone is approached. Since the extent of the dynamic meniscus is finite, one might assure that the value of this integral remains finite for any value of $\mathrm{Ca}$; on the other hand, as $\mathrm{Ca} \rightarrow 0$ the value of $\alpha$ must go to infinity and consequently $\beta / \alpha \rightarrow 1$. This result can be corroborated with the values of Tables I and II; we can check that $\beta / \alpha$ is 6.51 when $C a=10^{-7}, 1.2052$ when $C a=10^{-8}$, and 1.018 when $C a=10^{-9}$ and $h_{\infty}=2.0579 \times 10^{-8}$ (these last values are not shown in Table II) and the correctness of Eq. (14) is confirmed.

With the purpose of performing a more detailed analysis on the interaction between the main forces involved in this flow system, Eq. (6) was redefined using capillary length as the characteristic length parameter. The resulting equation has two dimensionless parameters, the ratio of molecular 


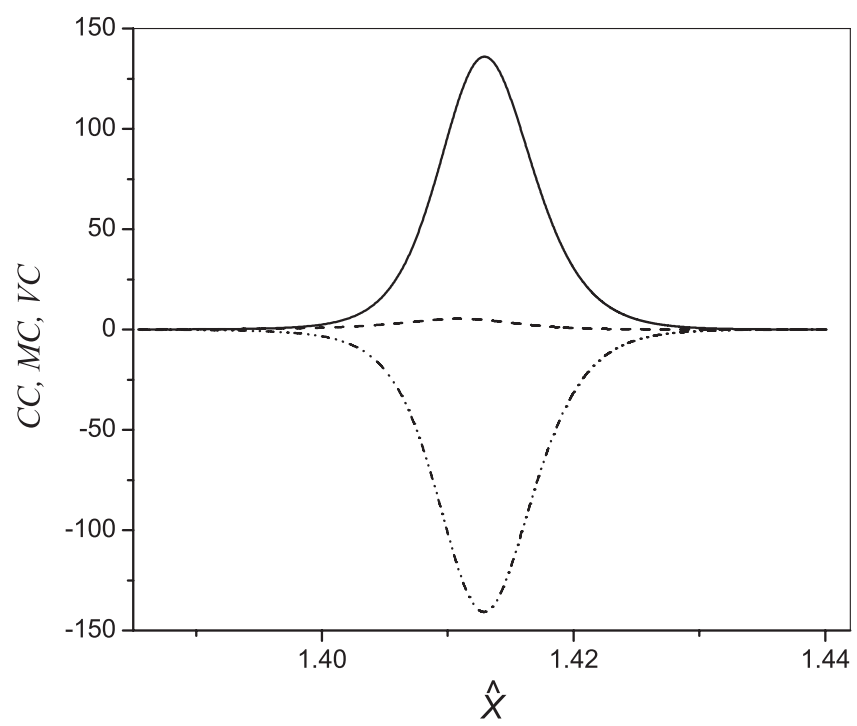

FIG. 4. Relative magnitude of capillary (- - CC), molecular (- MC) and viscous (- - VC) components [Eq. (18)] along the dynamic meniscus region for $\mathrm{Ca}=10^{-8}$ and $\mathrm{A}=6.13 \times 10^{-13}$.

and capillary lengths, $\left(h_{m}^{2} / L_{C}^{2}\right)$, and Capillary number

$$
\underbrace{\frac{d^{3} \hat{H}}{d \hat{X}^{3}}}_{\text {Capillary component }} \underbrace{-\frac{3 \mathrm{~A}}{\hat{H}^{4}} \frac{d \hat{H}}{d \hat{X}}}_{\text {Molecular component }}+\underbrace{3 C a\left(\frac{\hat{H}-\hat{H}_{\infty}}{\hat{H}^{3}}\right)}_{\text {Viscous component }}=0 ; \quad\left\{\begin{array}{l}
\hat{H}=\frac{h}{L_{C}} \\
\hat{X}=\frac{x}{L_{C}}
\end{array} .\right.
$$

The first term is the gradient of the curvature, the second the gradient of the disjoining pressure, and the third term, the viscous component, is the viscous force per unit volume. The relative magnitudes of these terms are shown in Figure 4 where their values are plotted as a function of dimensionless position $\hat{X}$ along the dynamic meniscus. Interestingly, within this region gradients of capillary and molecular forces are relatively large and of different sign while values of the viscous component are comparatively small. This behavior is similar to the evolution of capillary and molecular terms in a static liquid puddle, where a transition region is clearly defined in a region of space where capillary and molecular forces are of similar magnitude but different sign (Diaz Martin et al. ${ }^{14}$ ). Since the molecular term includes the first derivative of the film thickness with respect to $\hat{X}$, when the film approaches the constant film thickness, disjoining pressure remains nearly constant but film slope vanishes and so does the molecular component. Obviously the capillary term, being affected by the curvature of the interface vanishes just as rapidly. Notice there is also a small maximum value of the viscous component within the dynamic region, slightly upstream to the minimum of the capillary term. Indeed, this is the region where viscous stresses are larger due to larger velocity gradients within the film.

To illustrate in more detail the behavior of the capillary, molecular, and viscous components, computed values of these terms at the point of maximum gradient of molecular forces are shown in Figure 5, for a constant value of $\mathrm{A}=6.13 \times 10^{-13}$ and increasing Capillary numbers. Notice that for small Capillary numbers, the maximum values of the gradients of capillary and molecular forces follow the same trend shown in Figure 4, i.e., they cancel each other out. However, for larger values of Capillary numbers, the molecular component becomes increasingly irrelevant and the viscous component and the gradient of capillary forces equilibrate as one would expect to find within the $L \& L^{1}$ regime. Terms involving Capillary and molecular forces dominate the balance of forces in the dynamic meniscus region for very small values of Capillary numbers. For larger values of Capillary number, film thicknesses are everywhere too large, $h(x)>10^{-7} \mathrm{~m}$, for molecular forces to have any sensible effect on the flow field. 


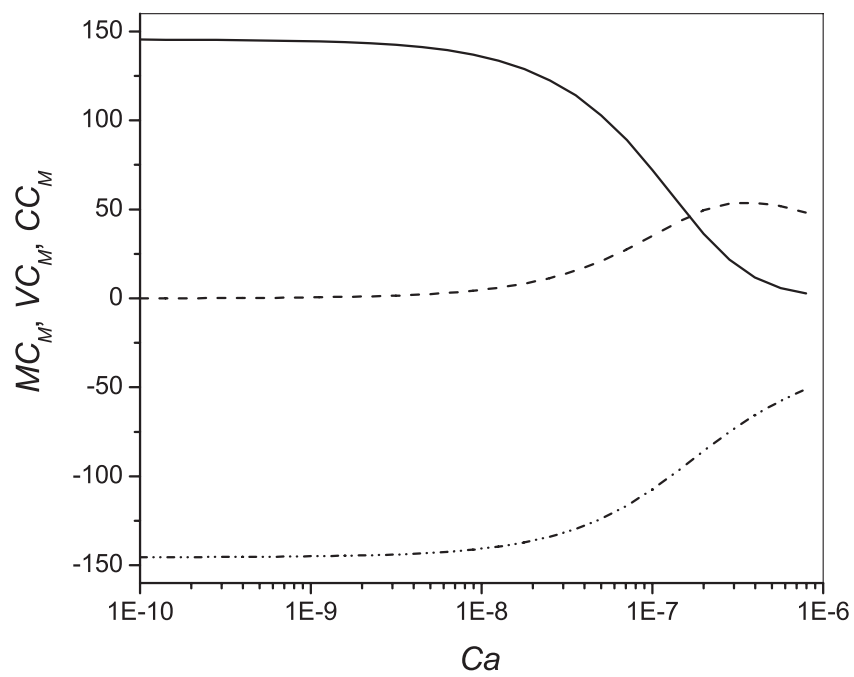

FIG. 5. Comparison of capillary $\left(-\cdots \mathrm{CC}_{\mathrm{M}}\right)$, molecular $\left(-\mathrm{MC}_{\mathrm{M}}\right)$ and viscous $\left(--\mathrm{VC}_{\mathrm{M}}\right)$ terms [Eq. (18)] at the point of maximum value of molecular forces along the film length. The maximum values are shown as a function of Capillary numbers for a constant value of $\mathrm{A}=6.13 \times 10^{-13}$.

One of the advantages of numerical simulations is the ability to visualize the evolution of the characteristic variables as a function of position along the flow field. A very interesting picture of the smooth matching between the static and dynamic meniscus regions is shown in Figure 6.

Figure 6 shows the static meniscus described by the solution of the Young-Laplace equation between the liquid pool and the point of matching. The line to the right of the matching points (indicated with $(\mathrm{x})$ for $\mathrm{A}=10^{-15}$, and $(*)$ for $\mathrm{A}=10^{-12}$ ) is the numerical solution to the film evolution equation. The inset in Figure 6 is a blowup of the dynamic meniscus region where film thicknesses are shown for two different values of A $\left(10^{-15}\right.$ and $\left.10^{-12}\right)$. Actually, the film profile shown for $\mathrm{A}=10^{-15}$ exactly coincides with $\mathrm{L} \& \mathrm{~L}^{1}$ predictions since the molecular forces are not strong enough when $\mathrm{Ca}=10^{-6}$ (see Figure 3); however, if the molecular forces become stronger $\left(\mathrm{A}=10^{-12}\right)$, their influence is evident and predictions show that not only the final film thickness is augmented but also most of the film profile along the dynamic meniscus moves towards larger

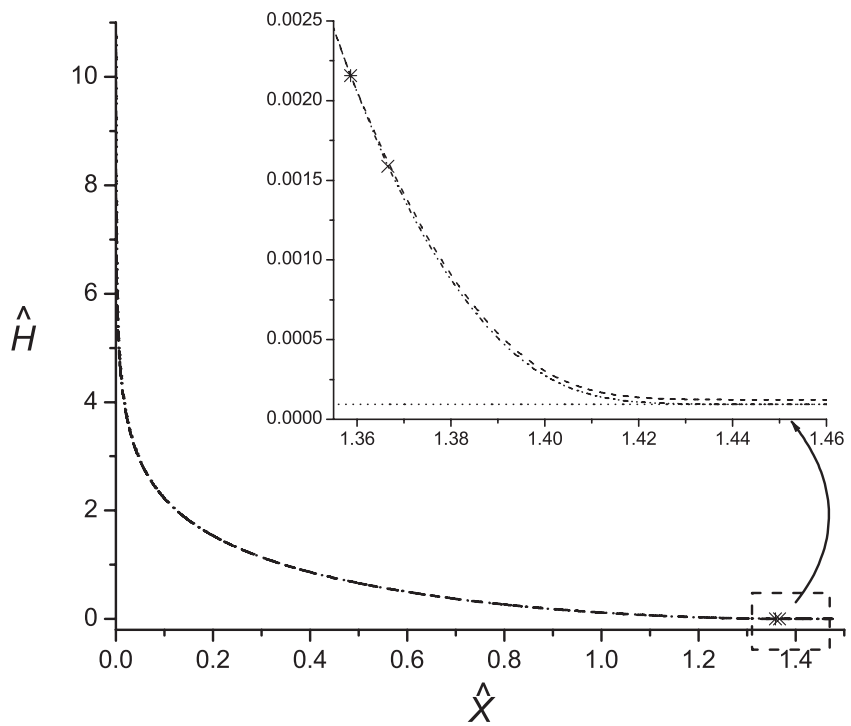

FIG. 6. Dimensionless film thickness, $\hat{H}$, versus dimensionless longitudinal variable, $\hat{X}$ for $C a=10^{-6}$, and A equal to $10^{-12}(--)$, and $10^{-15}(-\cdots)$. In the inset, the dotted line is $\hat{H}_{\infty}$ of the $L \& L^{1}$ solution. 
values of $\hat{H}$. As one approaches the point where the dynamic meniscus matches the static one, the film thickness increases and the action of the molecular forces become ineffective; therefore, the film profile merges with the $\mathrm{L} \& \mathrm{~L}^{1}$ film profile before the matching point is reached.

Another interesting feature observed in the inset of Figure 6 is the stretching of the dynamic meniscus; indeed, when the molecular forces act $\left(\mathrm{A}=10^{-12}\right)$, it becomes considerably larger than the case of $\mathrm{A}=10^{-15}$, where they are too weak to be effective for $\mathrm{Ca}=10^{-6}$.

The smooth matching is not observed if we analyze the evolution of the curvature and disjoining pressure in both the dynamic and static regions. Curvature for the static meniscus region is represented in terms of the augmented Young-Laplace equation, Eq. (7). We include molecular forces in Eq. (19) even when we have already shown in Sec. III that molecular forces are negligible in the static meniscus region where the dominant terms are capillarity and gravity,

$$
\hat{P}_{\text {static }}(\hat{X})=-\hat{X}-\frac{\mathrm{A}}{\hat{H}^{3}} .
$$

In the static meniscus region, it is clear that curvature is corresponded by a static pressure term, $\hat{P}_{\text {static }}(\hat{X})$, made up by the contribution of gravity and disjoining pressure, although the last is negligible. Integration across the film thickness to develop the film evolution equation is accomplished by introducing an interface curvature term, representing pressure other than disjoining pressure. The rigorous physical meaning of fluid pressure is lost but we might define by analogy a dynamic pressure term, $\hat{P}_{d y n}(\hat{X})$, similar to the static term, representing hydrodynamic pressure plus disjoining pressure. The dynamic pressure term is defined by integration of the dimensionless film evolution equation, Eq. (18), where gravity and viscous forces are neglected, and curvature and disjoining pressure prevail

$$
\hat{P}_{d y n}\left(\hat{X}_{L}\right)-\hat{P}_{d y n}(\hat{X})=\int_{\hat{X}_{L}}^{\hat{X}}\left\{\frac{d^{3} \hat{H}^{3}}{d \hat{X}^{3}}-\frac{3 \mathrm{~A}}{\hat{H}^{4}} \frac{d \hat{H}}{d \hat{X}}\right\} d \hat{X} \cong\left[\frac{d^{2} \hat{H}}{d \hat{X}^{2}}+\frac{\mathrm{A}}{\hat{H}^{3}}\right]-\sqrt{2}
$$

In the presence of a gravity field, hydrostatic pressure and interface curvature are linear functions of elevation, as indicated by Eqs. (7) and (19). Within the dynamic meniscus region, when molecular forces are increasingly important, there is a transition region where disjoining pressure becomes increasingly large and capillary and molecular forces make up the force balance with a small contribution of viscosity. Figure 7 shows dimensionless pressure terms, $\hat{P}_{\text {static }}(\hat{X})$ and $\hat{P}_{d y n}(\hat{X})$, i.e., the terms shown to the right hand side of Eqs. (19) and (20), as a function of elevation along the solid surface. The straight line on the left side of Fig. 7 shows the linear variation of pressure with elevation in the static meniscus. The S-shaped curve on the right hand side of Fig. 7 represents the variation of $\hat{P}_{d y n}(\hat{X})$ within the dynamic meniscus region where it sharply increases up to a constant value within a very small region. There are two important issues that beg to point out. First, rather surprisingly, matching of curvature between static and dynamic pressure, $\hat{P}_{\text {static }}(\hat{X})$ and $\hat{P}_{d y n}(\hat{X})$, show a small, but distinctive discontinuity. This discontinuity can be traced back to the approximation introduced by neglecting the first derivative of the film thickness, $d h / d x \ll 1$, from the curvature of the interface. Indeed, the expression for curvature of the static meniscus described by Eq. (7) is exact, while the description of curvature introduced with the film evolution equation, Eq. (18), is only approximate. Second, even when the matching values are unaffected, sharp variations in pressure inside the film take place to catch up with atmospheric pressure and a growing disjoining pressure. Dynamic pressure rapidly accommodates to reach the level of disjoining pressure in the thin film downstream and is obviously different for different values of the molecular constant, A. It is the evolution of dynamic pressure, $\hat{P}_{d y n}(\hat{X})$, the mechanism that acts as a metering device and limits the amount of liquid carried by the moving solid. Indeed, the measurable value of disjoining pressure within the constant film thickness region, $\hat{P}_{d y n}(\hat{X} \rightarrow \infty)$ shown in Figure 7 , is enough to justify a film thickness behavior departing from traditional $L \& \mathrm{~L}^{1}$ theory. More important, and consistent with the theory outlined in this paper, molecular forces only affect the dynamic meniscus region. 


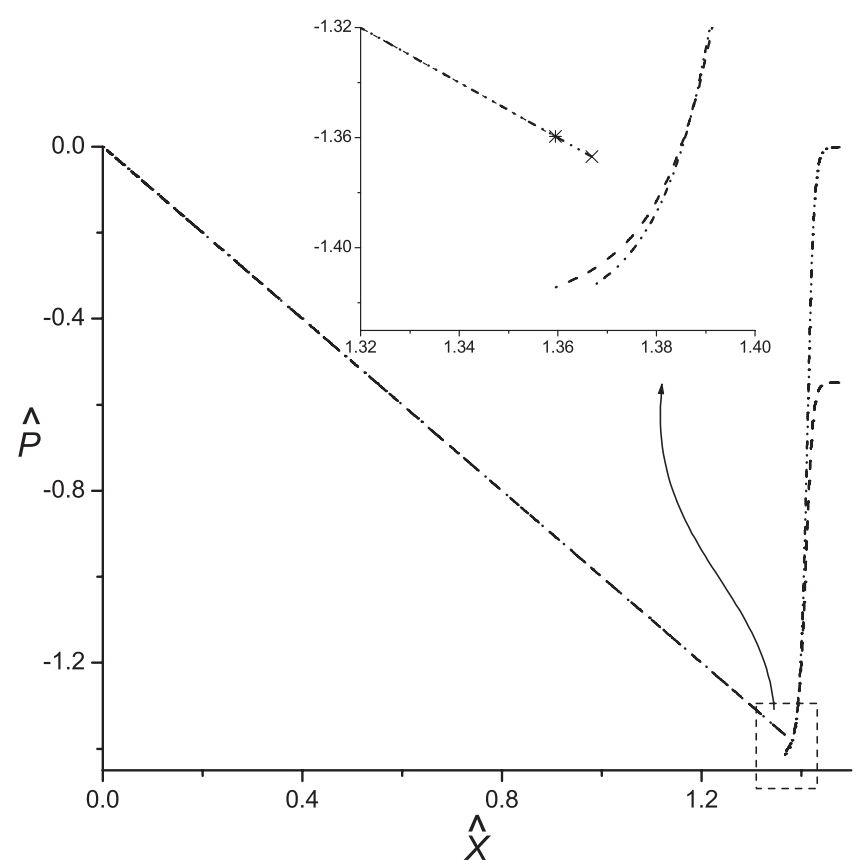

FIG. 7. Dimensionless pressure versus dimensionless elevation for $\mathrm{Ca}=10^{-6}$. The small square shows a detail of the region where static and dynamic menisci meet. The augmented terms on the right hand side are for values of A equal to $10^{-12}\left(-{ }^{-}\right)$ and $10^{-15}(-\cdots)$.

\section{CONCLUSIONS}

The $L \& L^{1}$ approach is based on two basic assumptions. The first assumption prescribes that the shape of the interface in the static meniscus region is described by the augmented Young-Laplace equation. This assumption was demonstrated to be valid (Sec. II) in the presence of non-polar molecular forces. For very small Capillary numbers, even when the dimensionless film thickness no longer complies with Eq. (2), the shape of the gas/liquid interface in the static meniscus region is described by the augmented Young-Laplace equation within a very small error. Similar conclusions were reached by Vannozzi ${ }^{11}$ using a theoretical Debye-Huckel electrostatic potential. The second assumption implies that at the point of matching the contact angle is vanishingly small and the film thickness is at least an order of magnitude larger than the range of the molecular forces. If the matching constant angle becomes larger than zero, it could be within the so-called dynamic dewetting regime, not within the film drag out regime.

For Capillary numbers about $10^{-6}$ or smaller, there is a large departure from the $\mathrm{L}_{\mathrm{L}} \mathrm{L}^{1}$ solution, Eq. (2). The departure consists of a flattening-out of the curve representing dimensionless film thickness versus Capillary numbers for constant values of molecular force parameters. This physical behavior was experimentally observed on fiber coating by Quére et al. ${ }^{6}$ Within the $\mathrm{L}_{\mathrm{L}} \mathrm{L}^{1}$ regime, dimensionless film thickness, $\hat{H}_{\infty}$, increases with the $2 / 3$ power of Capillary number $\hat{H}_{\infty} \sim C a^{2 / 3}$, i.e., the larger the wall velocity the larger the film thickness. Within the lower Capillary number regime, dimensionless film thickness becomes a constant and it is only function of molecular and capillary lengths, $\hat{H}_{\infty}(\mathrm{A}) \sim$ const.

Molecular forces result in sensible changes in film thickness profiles along a significant part of the dynamic meniscus, even when film thickness at the point of matching is too large to be affected by molecular forces; this result indicates that molecular forces are important within the dynamic meniscus. Another important effect of molecular forces is the stretching detected in the dynamic meniscus when the parameter A is augmented.

Regardless of the value of A, film matching is smooth as indicated by the smooth transition in Figure 6. Moreover, the film thickness at the point of matching, $h\left(x_{L}\right)$, is over an order of magnitude larger than film thickness downstream, $h_{\infty}$. Since the curvature of the interface was simplified, 
$d h / d x \ll 1$, matching static and dynamic pressures is not as smooth as the matching of film thickness and a small jump in the pressure field is observed. However, the pseudo-pressure term within the dynamic meniscus region, $\hat{P}_{d y n}(\hat{X})$, shows differences in evolution as well as in the limiting value, $\hat{P}_{d y n}(\hat{X} \rightarrow \infty)$, as seen in Figure 7 . This limiting value is determined by disjoining pressure in the constant film region, and it is responsible for final film thicknesses $\left(\hat{H}_{\infty}\right)$ different from $\mathrm{L}_{\mathrm{L}} \mathrm{L}^{1}$ results.

\section{ACKNOWLEDGMENTS}

We are grateful to CONICET and Universidad Nacional del Litoral for the support provided, and to the ANPCyT of Argentina for its grant in aid through the PICT raíces 00212.

\footnotetext{
${ }^{1}$ L. Landau and B. Levich, "Dragging of a liquid by a moving plate," Acta Physicochim. USSR 17, 42-54 (1942). Note: There is a brief note at the bottom of page 46 of Landau and Levich (1942) paper stating: "One of us (B. Levich) has been kindly informed by B. V. Derjaguin that he was first to obtain this equation [Nota bene: Equation 1.5]. However, B. V. Derjaguin has failed to derive from it any particular conclusions concerning the thickness of the film carried along." Derjaguin's paper was published in the same journal, but in volume XX, in 1945. There is a note at the bottom of the first page of the article claiming: "This derivation was presented at a colloquium in the Research Institute of Cinematography and Photography in 1939-1940 and at a colloquium in the Laboratory of Thin Films of the Institute of Colloid and Electrochemistry in 1940. Publication was delayed due to the discovery of divergences from experiment, the explanation for which was found later. The experimental data available today fully confirm the theory including the numerical coefficients. These data will shortly be published."

${ }^{2}$ J. J. van Rossum, "Viscous lifting and drainage of liquids," Appl. Sci. Res. 7, 121-144 (1958).

${ }^{3}$ C. Y. Lee and J. A. Tallmadge, "Meniscus shapes in withdrawal of flat sheets from liquid baths: Dynamic profile data at low Capillary numbers," Ind. Eng. Chem. Fundam. 13, 356-360 (1974).

${ }^{4}$ D. A. White and J. A. Tallmadge, "Theory of drag out of liquids on flat plates," Chem. Eng. Sci. 20, 33-37 (1965).

${ }^{5}$ S. D. R. Wilson, “The drag out problem in film coating theory," J. Eng. Math. 16, 209-221 (1982).

${ }^{6}$ D. Quéré, J. Di Meglio, and F. Brochard-Wyart, "Making van der Waals films on fibers," Europhys. Lett. 10, 335-340 (1989).

${ }^{7}$ R. L. Cerro and L. E. Scriven, "Rapid free surface film flows: An integral approach,” Ind. Eng. Chem. Fundam. 19, 40-50 (1980).

${ }^{8}$ M. E. Diaz Martin, "Physicochemistry and hydrodynamics of Langmuir-Blodgett film depositions," Ph.D. dissertation, University of Alabama in Huntsville, 2004.

${ }^{9}$ G. F. Teletzke, "Thin liquid films, molecular theory and hydrodynamic implications," Ph.D. dissertation, University of Minnesota, 1983.

${ }^{10}$ R. Krechetnikov and G. M. Homsy, "Dip coating in the presence of a substrate-liquid interaction potential," Phys. Fluids 17, 102105 (2005).

${ }^{11}$ C. Vannozzi, "Dip coating with an interaction potential normal to the substrate," Phys. Fluids 24, 022107 (2012).

${ }^{12}$ R. Schunk, A. J. Hurd, and C. J. Brinker, "Free-meniscus coating processes," in Liquid Film Coating, edited by S. F. Kistler and P. M. Schweitzer (Chapman and Hall, New York, 1996), Chap. 13.

${ }^{13}$ B. G. Higgins and L. E. Scriven, "Interfacial shape and evolution equations for liquid films and other viscocapillary flows," Ind. Eng. Chem. Fundam. 18, 208-215 (1979).

${ }^{14}$ M. E. Diaz Martin, J. Fuentes, R. L. Cerro, and M. D. Savage, "An analytic solution for a partially wetting puddle and the location of the static contact angle," J. Colloid Interface Sci. 348, 232-239 (2010).

${ }^{15}$ J. E. McNutt and G. M. Andes, "Relationship of the contact angle to interfacial energies," J. Chem. Phys. 30, 1300-1303 (1959).

${ }^{16}$ M. E. Diaz Martin, J. Fuentes, R. L. Cerro, and M. D. Savage, "Hysteresis during contact angle measurement," J. Colloid Interface Sci. 343, 574-583 (2010).

${ }^{17}$ D. M. Campana, S. Ubal, M. D. Giavedoni, and F. A. Saita, "Numerical prediction of the film thickening due to surfactants in the Landau-Levich problem," Phys. Fluids 22, 032103 (2010).
} 